\title{
MODELAÇÃO BIM A PARTIR DOS REQUISITOS DE INFORMAÇÃO
}

\author{
(1) ISEL, Lisboa \\ (2) LNEC, Lisboa \\ (3) LNEC, Lisboa \\ (4) ISEL, Lisboa
}

Lorena Luedy $^{(1)}$, Paula Couto ${ }^{(2)}$, Maria João Falcão ${ }^{(3)}$, João Hormigo ${ }^{(4)}$

\begin{abstract}
Resumo
O Building Information Modelling (BIM), surge como uma das grandes revoluções da Engenharia Civil moderna. A metodologia BIM reúne processos, políticas e tecnologias que viabilizam a construção colaborativa num ambiente virtual, assentando a sua principal diferença, em relação aos processos tradicionais, na gestão da informação contida nos modelos tridimensionais desde o momento da conceção, passando pela construção e incluindo até a manutenção e reabilitação dos empreendimentos de construção.

O termo Nível de Informação Necessária surgiu em 2018 para auxiliar na definição da extensão e do nível de detalhe necessários para que cada ação proveniente do projeto tenha o conteúdo essencial para sua execução. Este processo de definição dos requisitos de informação deve estar vinculado à necessidade de partilha e à funcionalidade da informação de forma a evitar excesso de dados e de trabalho no desenvolvimento dos modelos BIM.

Neste contexto, o trabalho apresentado pretende tornar tangível, para engenheiros, arquitetos e outros intervenientes no processo, como os requisitos de informação são aplicados aos modelos BIM. No seu início, é apresentado um breve estado da arte sobre a metodologia BIM e quais são os requisitos de informação contidos nas normas de relevância mundial.

O caso de estudo consiste num espaço privado de lazer localizado na zona de Lisboa. A primeira etapa desenvolveu os requisitos de informação necessários para os modelos BIM em conformidade com as fases de estudo prévio, de projeto de execução e de modelo de exploração. Esta última fase compreende os processos de manutenção, operação e reabilitação do empreendimento. Posteriormente, apresentam-se os modelos desenvolvidos para cada uma das referidas fases do processo a partir dos requisitos de informação estabelecidos. Para terminar faz-se a análise dos resultados obtidos no caso de estudo, sendo perspetivados e propostos trabalhos futuros.
\end{abstract}

\section{Introdução}

A metodologia BIM é um vetor da revolução e reestruturação dos processos já praticados no setor de Arquitetura, Engenharia, Construção e Operação (AECO). Nessa transformação, se 
torna necessário compreender como gerenciar os requisitos de informações nos modelos. Uma das principais mudanças se apresenta na contratação de serviços, pois nos projetos em BIM há muitos fatores a serem discutidos em relação à qualidade e quantidade de informações para definir o escopo do próprio projeto $[1,2]$.

Em 2018, o conceito de Nível de Informação Necessária foi publicado na ISO 19650-1 e na ISO 19650-2, promovendo uma solução para estabelecer esses requisitos de informações de contratação e gestão do projeto. Esse cenário de um conceito recente e da falta de pesquisas na área, torna urgente o desenvolvimento de estudos sobre o Nível de Informação Necessário para tornar esse conceito claro e prático para o setor AECO $[3,4]$.

O presente estudo aparece em uma conexão entre Portugal e Brasil, países em intensa implementação do BIM. Além disso, tem como objetivo analisar o conteúdo existente sobre o assunto, produzir uma estrutura de requisitos de informação e validá-la através de um caso de estudo.

\section{Estado da arte}

O Building Information Modelling (BIM) é um símbolo da inovação na indústria da construção, combinando tecnologia, processo e política para promover o projeto, a construção e a exploração digital no ambiente virtual. Esta metodologia promove o trabalho colaborativo entre os intervenientes em todo o ciclo de vida de qualquer edifício ou infraestrutura. Além disso, permite a digitalização, simulação e otimização dos processos construtivos alinhadas aos componentes paramétricos, reforçando o impacto da metodologia BIM no setor $[1,3,5]$.

Quando comparado aos projetos tradicionais bidimensionais, identifica-se que o uso do BIM viabiliza que os problemas sejam identificados, e tenham suas soluções adotadas, em fases anteriores do projeto. $\mathrm{O}$ grande benefício é que, o quanto antes são identificados os problemas, há mais capacidade de modificar o projeto, e essas mudanças custam menos. Em uma expansão global, um estudo recente previu uma economia de 15 a $25 \%$ no mercado de infraestrutura por meio da implementação do BIM [6,7].

Portugal e Brasil são países que estão avançando na implementação do BIM. Ambos já possuem uma Comissão Técnica de Normalização e preveem um impacto positivo na sua economia. Portugal avança na utilização do BIM no meio académico e industrial, juntamente com o avanço tecnológico. Na mesma direção, o Brasil já publicou sua Estratégia Nacional de Disseminação de BIM e prevê um aumento de dez vezes a participação de empresas que usam o BIM no produto nacional bruto da construção $[8,9,10]$.

O Nível de Desenvolvimento (LOD) é o principal recurso atualmente utilizado para alinhamento dos parâmetros do BIM entre as partes interessadas e para evitar excesso de trabalho e desperdício de informações. Definido pela primeira vez em 2009 nos Estados Unidos, o LOD é uma métrica de seis etapas usada para descrever as informações e a precisão gráfica que cada elemento precisa ter no projeto e construção. Essa especificação é conhecida pela comunidade de utilizadores do BIM, no entanto, possui significados e níveis diferentes por todo o mundo [11, 12].

As normas ISO 19650-1 e ISO 19650-2, publicadas em 2018, descrevem o novo conceito de Nível de Informação Necessária, a fim de desenvolver um modo mais adaptável e digital para os requisitos de informações dos modelos BIM. O Nível de Informação Necessária é a estrutura usada para definir a extensão e a granularidade das informações, estabelecendo as condições 
mínimas necessárias para definir, partilhar, executar, controlar e verificar cada informação [12, $3,4,13]$.

A Tabela 1 resume os aspetos relevantes do tópico nas normas europeias ISO 12006-2 (2015), ISO 19650 (2018), BS 1192-4 (2014). Também são considerados no quadro, guias relevantes usados nos Estados Unidos da América, Europa e Brasil: Fórum BIM e Caderno BIM de Santa Catarina $[11,3,4,14,15,16]$.

Tabela 1: Aspetos dos Requisitos de Informação em normas e guias

\begin{tabular}{|c|c|}
\hline Documento & Aspetos relevantes \\
\hline ISO 12006-2 (2015) & Sistemas de classificação; tipos de propriedades \\
\hline ISO 19650-1 (2018) & $\begin{array}{l}\text { Gerenciamento de informação; Aplicabilidade dos Requisitos de } \\
\text { Informação; Condições para partilhar informação em um ambiente } \\
\text { de dados comum }\end{array}$ \\
\hline ISO 19650-2 (2018) & $\begin{array}{l}\text { Gerenciamento de informação; definição, aplicação, partilha e } \\
\text { verificação do Nível de Informação Necessária }\end{array}$ \\
\hline BS 1192-4 (2014) & Estrutura de partilha de informação \\
\hline BIM Forum (2018) & Nível de Desenvolvimento; Nível de Detalhe; Nível de Informação \\
\hline $\begin{array}{l}\text { Caderno BIM de Santa } \\
\text { Catarina (2014) }\end{array}$ & $\begin{array}{l}\text { Nível de Desenvolvimento para cada fase de projeto; Propriedades } \\
\text { dos elementos }\end{array}$ \\
\hline
\end{tabular}

\section{Estrutura para os Requisitos de Informação em modelos BIM}

O trabalho proposto visa aplicar os conceitos e parâmetros de requisitos de informação aos modelos BIM para a definição de uma estrutura básica de referência para utilizadores iniciantes do BIM no setor AECO. Inicialmente, foram definidas as fases do projeto, disciplinas e lista de informações a serem analisadas. De acordo com os padrões e guias descritos anteriormente, todos esses aspetos foram relacionados à construção da estrutura, considerando quais informações são necessárias para a execução da fase do projeto. Os parâmetros para personalizar essa estrutura para a realidade de cada projeto são finalmente indicados.

A Portaria n. ${ }^{\circ} 701-\mathrm{H} / 2008$ de Portugal, que declara as fases do projeto e sua entregas esperadas, foi a base principal para estabelecer as fases do projeto. O Programa Base é uma fase que afirma apenas que o executor usará o BIM, não tendo nenhuma definição de informações ou requisitos geométricos, por isso que essa etapa não faz parte do caso de estudo. Posteriormente, o projeto passa pelo Programa Base, Estudo Prévio, Anteprojeto e Projeto de Execução quando o BIM pode ser útil e lucrativo. Por outro lado, as Telas Finais e a Exploração são contextos após a construção, que serão adicionadas nesta análise devido à sua relevância para os utilizadores do BIM. As disciplinas adotadas neste caso de estudo são as mais comuns nos projetos de edifícios e infraestrutura nos dois países em questão: Arquitetura e Estrutura. Adicionalmente, foi incluída a disciplina de Sistemas para representar como os projetos complementares são aplicados [17].

A lista principal de informações não gráficas foi baseada e simplificada pelo Caderno BIM de Santa Catarina. O complemento desta lista foi dado pela ISO 12006-2, que esclarece o conceito de Categoria, uma propriedade essencial ao Estudo Prévio. Para atender à Fase de Exploração, 
a informação "Dados para operação e manutenção" foi adicionada à lista. O Caderno BIM de Santa Catarina apresenta exemplos de quais informações são necessárias para cada LOD e relaciona o LOD com as fases do projeto. Utilizando isso como base principal para combinar as informações e considerando quais informações são indispensáveis para a execução, controle e verificação de cada fase do projeto, a estrutura foi desenvolvida como exemplo na Tabela 2 $[14,16]$.

Tabela 2: Requisitos de informação para modelos BIM

\begin{tabular}{|c|c|c|c|c|c|}
\hline \multirow[b]{2}{*}{ Disciplina | Informação } & \multicolumn{3}{|c|}{ Fase do projeto } & \multicolumn{2}{|c|}{ Pós-obra } \\
\hline & $\begin{array}{l}\text { Estudo } \\
\text { Prévio }\end{array}$ & Anteprojeto & $\begin{array}{l}\text { Projeto de } \\
\text { Execução }\end{array}$ & $\begin{array}{l}\text { Telas } \\
\text { finais }\end{array}$ & Exploração \\
\hline \multicolumn{6}{|c|}{ 1. Arquitetura } \\
\hline Categoria & $\mathrm{x}$ & $\mathrm{x}$ & $\mathrm{x}$ & $\mathrm{x}$ & $\mathrm{x}$ \\
\hline Tipo & & $\mathrm{x}$ & $\mathrm{x}$ & $\mathrm{x}$ & $\mathrm{x}$ \\
\hline Dimensão & $\mathrm{x}$ & $\mathrm{x}$ & $\mathrm{x}$ & $\mathrm{x}$ & $\mathrm{x}$ \\
\hline Localização & $\mathrm{x}$ & $\mathrm{x}$ & $\mathrm{x}$ & $\mathrm{x}$ & $\mathrm{x}$ \\
\hline Material & & & $\mathrm{x}$ & $\mathrm{x}$ & $\mathrm{x}$ \\
\hline Fabricante & & & & $\mathrm{x}$ & $\mathrm{x}$ \\
\hline Resistividade & & & $\mathrm{x}$ & $\mathrm{x}$ & $\mathrm{x}$ \\
\hline $\begin{array}{l}\text { Dados para operação e } \\
\text { manutenção }\end{array}$ & & & & & $\mathrm{x}$ \\
\hline \multicolumn{6}{|c|}{ 2. Estruturas } \\
\hline Categoria & & $\mathrm{x}$ & $\mathrm{x}$ & $\mathrm{x}$ & $\mathrm{x}$ \\
\hline Tipo & & $\mathrm{x}$ & $\mathrm{x}$ & $\mathrm{x}$ & $\mathrm{x}$ \\
\hline Dimensão & & $\mathrm{x}$ & $\mathrm{x}$ & $\mathrm{x}$ & $\mathrm{x}$ \\
\hline Localização & & $\mathrm{x}$ & $\mathrm{x}$ & $\mathrm{x}$ & $\mathrm{x}$ \\
\hline Material & & & $\mathrm{x}$ & $\mathrm{x}$ & $\mathrm{x}$ \\
\hline Fabricante & & & & $\mathrm{x}$ & $\mathrm{x}$ \\
\hline Resistividade & & & $\mathrm{x}$ & $\mathrm{x}$ & $\mathrm{x}$ \\
\hline
\end{tabular}

Dados para operação e manutenção

\section{Sistemas}

\begin{tabular}{lllll}
\hline Categoria & $\mathrm{x}$ & $\mathrm{x}$ & $\mathrm{x}$ & $\mathrm{x}$ \\
\hline Tipo & $\mathrm{x}$ & $\mathrm{x}$ & $\mathrm{x}$ & $\mathrm{x}$ \\
\hline Dimensão & $\mathrm{x}$ & $\mathrm{x}$ & $\mathrm{x}$ & $\mathrm{x}$ \\
\hline Localização & $\mathrm{x}$ & $\mathrm{x}$ & $\mathrm{x}$ & $\mathrm{x}$ \\
\hline Material & & $\mathrm{x}$ & $\mathrm{x}$ & $\mathrm{x}$ \\
\hline Fabricante & & $\mathrm{x}$ & $\mathrm{x}$ \\
\hline Resistividade & $\mathrm{x}$ & $\mathrm{x}$ & $\mathrm{x}$ \\
\hline $\begin{array}{l}\text { Dados para operação e } \\
\text { manutenção }\end{array}$ & & & $\mathrm{x}$ \\
\hline
\end{tabular}


Vale ressaltar que esta é uma proposta para uma estrutura geral a ser utilizada como base para o planejamento, execução e controle dos requisitos de informação para modelos BIM. Para produzir um modelo fácil de entender e básico de utilizar, simplificações e combinações de conceitos foram realizados. O público-alvo deste estudo são arquitetos e engenheiros que estão a implementar o BIM nos seus projetos.

A partir do referencial generalista, é indicada, para cada informação, uma análise de sua adaptação à realidade do projeto e da empresa. As normas europeias ISO 19650-1 (2018) e ISO 19650-2 (2018) descrevem em detalhes os aspetos a serem considerados nesta análise dos requisitos de informações do modelo BIM. Os aspetos, que podem ser descritos como parâmetros de personalização (Figura 1), são: i) plano de trabalho do projeto; ii) propósito da informação; iii) ciclo de partilha de informações; iv) necessidades do cliente; v) decisões provenientes da informação; vi) qualidade da informação; vii)quantidade de informações; viii) requisitos geométricos; ix) requisitos alfanuméricos [3,4].

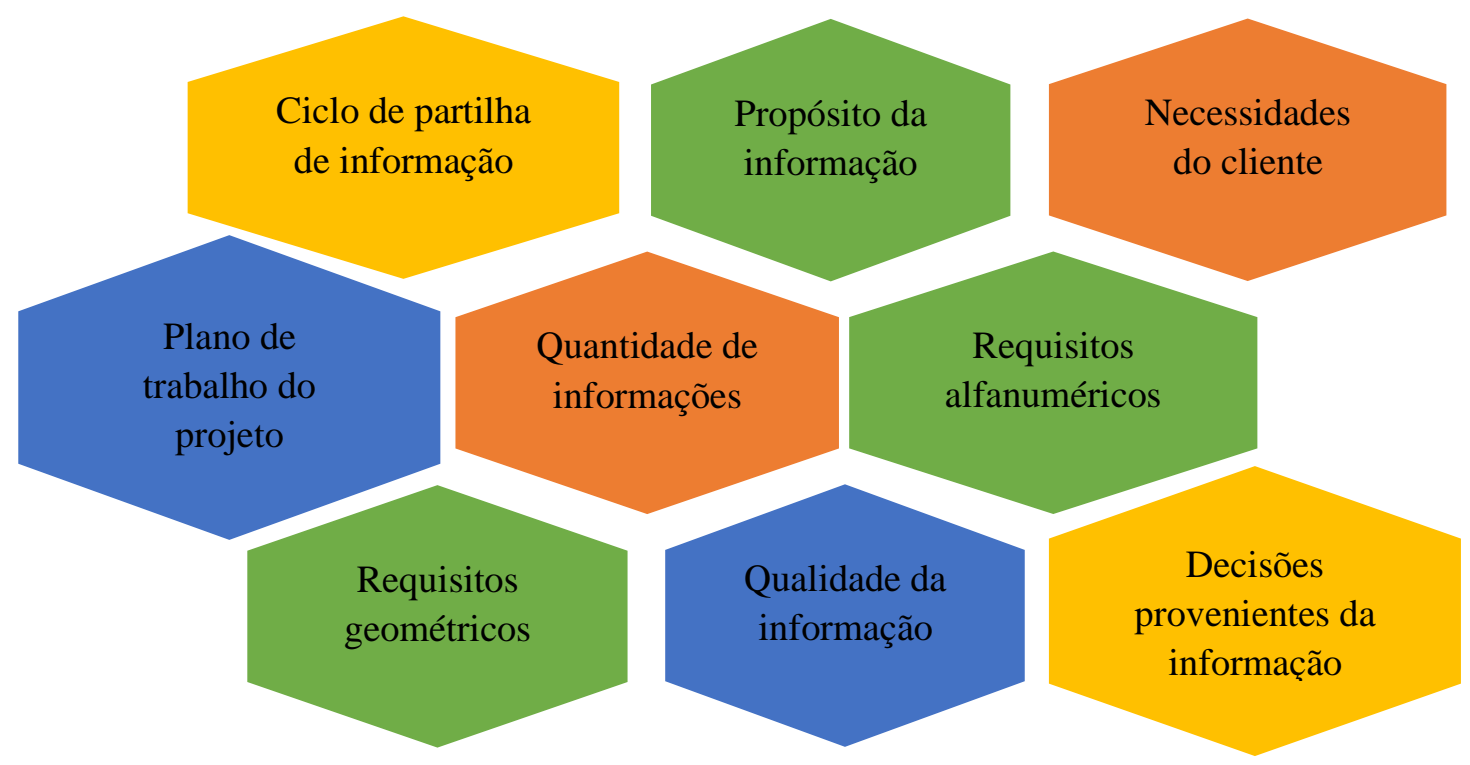

Figura 1: Parâmetros para personalização dos requisitos de informação para cada modelo BIM.

Posteriormente, a aplicabilidade da estrutura de Requisitos de Informação e os parâmetros de personalização foi avaliada num caso de estudo. $\mathrm{O}$ edifício analisado foi um cinema localizado num centro comercial em Lisboa. Os documentos utilizados para o processo de modelação em BIM foram os projetos tradicionais da fase de execução em 2D, fornecidos pela empresa SONAE Sierra, proprietária e gestora do ativo. A exceção foi o projeto de Estruturas fornecido já em BIM no formato IFC (Industry Foundation Classes).

\section{Resultados}

Considerando os projetos fornecidos, as necessidades do cliente e a quantidade de informações e trabalho necessários para analisar a estrutura proposta para o caso de estudo, a nova estrutura de requisitos de informação foi construída. As fases de projeto analisadas foram o estudo prévio, 
o projeto de execução e a exploração. Como tal, as especialidades modeladas foram a Arquitetura, as Estrutura e as Instalações, equipamentos e sistemas de águas e esgotos. A estrutura dos Requisitos de Informação para os modelos BIM foi adaptada pelos parâmetros personalizados ao caso de estudo (Tabela 3 ).

Tabela 3: Requisitos de informação para modelo BIM do caso de estudo

\begin{tabular}{|c|c|c|c|}
\hline \multirow[b]{2}{*}{ Disciplina | Informação } & \multicolumn{2}{|c|}{ Fase de projeto } & \multirow{2}{*}{$\begin{array}{c}\text { Pós-obra } \\
\text { Exploração }\end{array}$} \\
\hline & Estudo Prévio & $\begin{array}{l}\text { Projeto de } \\
\text { Execução }\end{array}$ & \\
\hline \multicolumn{4}{|c|}{ 1. Arquitetura } \\
\hline Categoria & $\mathrm{x}$ & $\mathrm{x}$ & $\mathrm{x}$ \\
\hline Tipo & & $\mathrm{x}$ & $\mathrm{x}$ \\
\hline Dimensão & $\mathrm{x}$ & $\mathrm{x}$ & $\mathrm{x}$ \\
\hline Localização & $\mathrm{x}$ & $\mathrm{x}$ & $\mathrm{x}$ \\
\hline Material & & $\mathrm{x}$ & $\mathrm{x}$ \\
\hline \multicolumn{4}{|l|}{ Fabricante } \\
\hline \multicolumn{4}{|l|}{ Resistividade } \\
\hline $\begin{array}{l}\text { Dados para operação e } \\
\text { manutenção }\end{array}$ & & & $\mathrm{x}$ \\
\hline \multicolumn{4}{|c|}{ 2. Estruturas } \\
\hline Categoria & & $\mathrm{x}$ & \\
\hline Tipo & & $\mathrm{x}$ & \\
\hline Dimensão & & $\mathrm{x}$ & \\
\hline Localização & & $\mathrm{x}$ & \\
\hline Material & & $\mathrm{x}$ & \\
\hline \multicolumn{4}{|l|}{ Fabricante } \\
\hline \multicolumn{4}{|l|}{ Resistividade } \\
\hline $\begin{array}{l}\text { Dados para operação e } \\
\text { manutenção }\end{array}$ & & & \\
\hline
\end{tabular}

3. Instalações, equipamentos e sistemas de águas e esgotos

\begin{tabular}{ll}
\hline Categoria & $\mathrm{x}$ \\
\hline Tipo & $\mathrm{x}$ \\
\hline Dimensão & $\mathrm{x}$ \\
\hline Localização & $\mathrm{x}$ \\
\hline Material & $\mathrm{x}$
\end{tabular}

Fabricante

Resistividade

Dados para operação e

manutenção 
O cliente não tinha necessidade das informações de algumas características, nomeadamente dados do Fabricante e da resistividade nos modelos; pelo que essa informação ficou apenas, nos documentos auxiliares. Além disso, o cliente necessitava do modelo de exploração simplificado, contendo apenas o projeto de arquitetura e alguns dados para operação e manutenção. No estudo prévio, o Quadro de Requisitos de Informações (Quadro 3) foi da maior utilidade para restringir quais as informações realmente necessárias para o projeto, evitando desperdício de dados e de trabalho. Nesta fase, apenas foi necessário modelar o volume e a categoria do projeto de arquitetura (Figura 2.a). O recurso "Mass \& Site" do programa Revit da Autodesk foi útil para modelar paredes, teto falso e cobertura. As escadas foram modeladas como uma rampa para dar a dimensão simplificada de volume necessária.

$\mathrm{Na}$ fase do Projeto de Execução (Figura 2.b), todos os projetos foram modelados e compatibilizados, sendo a importância da informação patente do Quadro 3 a de orientar a quantidade de informação que cada elemento requer, direcionar a pesquisa das informações no projeto original e ajudar com o fluxo de trabalho do projeto. Os benefícios do uso da metodologia BIM foram ressaltados durante a modelação, na medida em que foram identificados erros de projeto, inconsistências entre as plantas e cortes e falta de informações no projeto original em 2D, o que ressalta. Para verificar o princípio de interoperabilidade BIM, o modelo em IFC foi incluído no projeto de execução. A estrutura foi fundamental para verificar se o modelo em IFC possuía todas as informações necessárias para a execução dessa fase, funcionando como uma lista de verificação.

A última fase modelada foi a Exploração. Para isso, foram solicitadas à SONAE Sierra as informações necessárias para a operação e manutenção do ativo. O principal espaço considerado na fase de Manutenção está sob as arquibancadas (Fig. 2.c), uma área que é usada para estoque e é gerenciada pela empresa. A área de cinema é gerenciada pelo lojista e não será muito útil para o cliente nesse contexto pós-obra. No modelo de exploração, o Quadro 3 foi útil para entendimento de quais informações precisam ser excluídas ou adicionadas para a entrega do modelo simplificado solicitado pelo cliente.

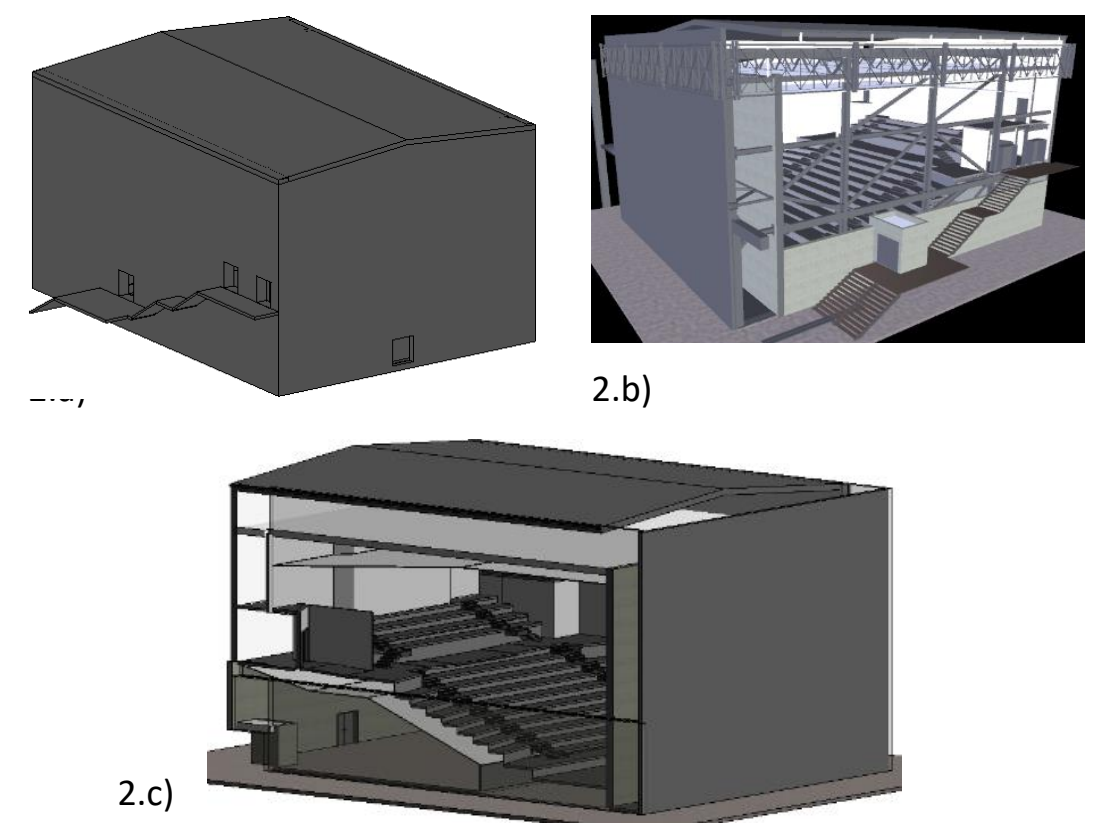

Figura 2: Estudo prévio, projeto de execução e modelo de exploração do caso de estudo. 


\section{Conclusões}

Como uma metodologia revolucionária na indústria da construção em todo o mundo, o BIM está a transformar os processos, do projeto à exploração e reabilitação, de edifícios e obras de infraestrutura. Tem-se como consequência uma nova necessidade de planear, definir, partilhar, operar e verificar os requisitos de informações dos modelos BIM. O conceito de Nível de Informação Necessária foi publicado em 2018 para solucionar esta situação de maneira digital, flexível, adaptável e útil.

A estrutura genérica proposta é baseada em diferentes padrões e guias, tendo exigido a comparação e a simplificação de conteúdo da documentação original. Esta estrutura visa ser a base para arquitetos e engenheiros, que estão a iniciar a implementação do BIM, entenderem e aplicarem os requisitos de informações nos seus projetos. Posteriormente, é necessário usar os parâmetros de personalização para adaptar a estrutura geral a cada realidade do projeto e da empresa.

A partir da aplicação da estrutura proposta ao caso de estudo conclui-se que a estrutura é útil e aplicável orientando todo o processo de modelação. Outro resultado importante foi que o projeto de estruturas, em formato IFC, foi verificado por meio da estrutura proposta, o que funciona como uma lista de verificação dos requisitos de informação, garantindo o princípio de interoperabilidade do BIM. Como todo o trabalho foi desenvolvido analisando em paralelo o cenário de Portugal e do Brasil, a proposta é aplicável a esses dois contextos. Para além disso, se os aspetos de outros países forem igualmente considerados nos parâmetros personalizados, a estrutura proposta torna-se extensível ao resto do mundo.

\section{Referências}

[1] EASTMAN, C. et al. Manual de BIM: um guia de modelação da informação da construção para arquitetos, engenheiros, gerentes, construtores e incorporadores. Porto Alegre: Bookman, 2014.

[2] CBIC. Implementação do BIM para Construtoras e Incorporadoras: Parte 5 - Formas de contratação BIM. Câmara Brasileira da Indústria da Construção, Brasília, 2016.

[3] INTERNATIONAL ORGANIZATION FOR STANDARDIZATION (ISO). ISO 196501: Organization and digitization of information about buildings and civil engineering works, including building information modelling (BIM) - Information management using building information modelling - Part 1: Concepts and principles. Geneva, Switzerland, 2018.

[4] INTERNATIONAL ORGANIZATION FOR STANDARDIZATION (ISO). ISO 196501: Organization and digitization of information about buildings and civil engineering works, including building information modelling (BIM) - Information management using building information modelling - Part 2: Delivery phase of the assets. Geneva, Switzerland, 2018.

[5] BIM DICTIONARY. BIM Dictionary platform. Disponível em: https://bimdictionary.com. Acesso em: 18 Ago. 2014.

[6] CBIC. Implementação do BIM para Construtoras e Incorporadoras: Parte 1 - Fundamentos BIM. Câmara Brasileira da Indústria da Construção, Brasília, 2016. 
[7] CT197-BIM. Guia da Contratação BIM. Comissão Técnica de Normalização BIM, Portugal, 2017.

[8] BIM COMMUNITY. Construção Civil em 2019: Maiores investimentos e projetos desafiadores. Disponível

em: https://www.bimcommunity.com/news/load/1096/construction-in-2019-majorinvestments-and-challenging-projects. Acesso em: 20 mar, 2019.

[9] HORE, A. et al. Global BIM Study Lessons for Ireland's BIM Programme. Construction IT Alliance Limited, Dublin, Ireland, 2017.

[10]BIM BR. Construção Inteligente. Ministério da Indústria, Comércio Exterior e Serviços, Brasil, 2018.

[11]BIM FORUM. Level of Development Specification Part I \& Commentary. AIA, United States, 2018.

[12]BOLPAGNI, M., CIRIBRINI, A. The Information Modeling and the Progression of DataDriven Projects. Milano, Italy, 2015.

[13]CAMILO, C. (2018). Qué normas y estándares BIM seguimos. MSI Studio. Disponível em: https://www.msistudio.com/que-normas-y-estandares-bim-seguimos. Acesso em: 03 mai, 2018.

[14]INTERNATIONAL ORGANIZATION FOR STANDARDIZATION (ISO). ISO 120062: Building construction - Organization of information about construction works - Part 2: Framework for classification. Geneva, Switzerland, 2015.

[15] BRITISH STANDARD (BS). BS 1192-4: Collaborative production of information. Part 4: Fulfilling employer's information exchange requirements using COBie - Code of practice. United Kingdom, 2014.

[16] GOVERNO DE SANTA CATARINA. Caderno BIM - Apresentação de Projetos de Edificações em BIM. Santa Catarina, Brasil, 2014.

[15] PORTARIA n. ${ }^{\circ}$ 701-H/2008. Ministério das Obras Públicas, Transportes e Comunicações, Portugal, 2008. 